
Jennifer Lippincott-Schwartz and George H. Patterson

Science 300, 87 (2003);

DOI: $10.1126 /$ science. 1082520

This copy is for your personal, non-commercial use only.

If you wish to distribute this article to others, you can order high-quality copies for your colleagues, clients, or customers by clicking here.

Permission to republish or repurpose articles or portions of articles can be obtained by following the guidelines here.

The following resources related to this article are available online at www.sciencemag.org (this information is current as of January 22, 2014 ):

Updated information and services, including high-resolution figures, can be found in the online version of this article at:

http://www.sciencemag.org/content/300/5616/87.full.html

Supporting Online Material can be found at:

http://www.sciencemag.org/content/suppl/2003/04/03/300.5616.87.DC1.html

A list of selected additional articles on the Science Web sites related to this article can be found at:

http://www.sciencemag.org/content/300/5616/87.full.html\#related

This article cites 56 articles, 19 of which can be accessed free:

http://www.sciencemag.org/content/300/5616/87.full.html\#ref-list-1

This article has been cited by 331 article(s) on the ISI Web of Science

This article has been cited by 62 articles hosted by HighWire Press; see:

http://www.sciencemag.org/content/300/5616/87.full.html\#related-urls

This article appears in the following subject collections:

Cell Biology

http://www.sciencemag.org/cgi/collection/cell_biol 


\title{
Development and Use of Fluorescent Protein Markers in Living Cells
}

\author{
Jennifer Lippincott-Schwartz* and George H. Patterson
}

\begin{abstract}
The ability to visualize, track, and quantify molecules and events in living cells with high spatial and temporal resolution is essential for understanding biological systems. Only recently has it become feasible to carry out these tasks due to the advent of fluorescent protein technology. Here, we trace the development of highly visible and minimally perturbing fluorescent proteins that, together with updated fluorescent imaging techniques, are providing unprecedented insights into the movement of proteins and their interactions with cellular components in living cells.
\end{abstract}

The development of fluorescent proteins as molecular tags over the past decade has spurred a revolution by allowing complex biochemical processes to be correlated with the functioning of proteins in living cells. Fluorescent proteins such as green fluorescent protein (GFP) from the jellyfish $\mathrm{Ae}$ quorea victoria (Fig. 1, A and B) and its variants can be fused to virtually any protein of interest to analyze protein geography, movement, and chemistry in living cells. As such, they have provided an important new approach for understanding protein function, filling an urgent need now that the genome sequence of many organisms is complete. The fluorescent proteins have been used as tools in numerous applications, including as minimally invasive markers to track and quantify individual or multiple protein species [reviewed in (1)], as probes to monitor protein-protein interactions [reviewed in $(2-$ $4)$ ], as photo-modulatable proteins to highlight and follow the fate of specific protein populations within a cell (5-7), and as biosensors to describe biological events and signals $(3,4)$. Results from these applications are providing profound new insights into protein function and cellular processes in the complex environment of the cell. Here, we trace the development of GFP and other intrinsically fluorescent proteins and discuss the kinetic microscopy methods of photobleaching and photoactivation used to monitor the appearance, location, movement, and degradation of proteins in living cells.

\section{Development of GFP and Other} Fluorescent Molecules as Protein Tags

GFP was first discovered as a companion protein to aequorin, the chemiluminescent protein from A. victoria. Upon purification

Cell Biology and Metabolism Branch, National Institute of Child Health and Human Development, National Institutes of Health, Bethesda, MD 20892, USA.

*To whom correspondence should be addressed. Email: jlippin@helix.nih.gov to homogeneity from the jellyfish, GFP by itself was found to fluoresce under excitation, requiring no substrates or coenzymes (2) (Fig. 1, A and B). After cloning of the GFP gene (8), expression in nonjellyfish organisms yielded a functional fluorescent protein (9). These breakthrough discoveries indicated that the gene for GFP contained all of the information necessary for proper synthesis of the fluorophore.

Mutagenesis studies of GFP yielded variants with improved folding and expression properties that stimulated GFP's widespread use as a fluorescent protein tag. A single amino acid substitution, $\mathrm{Ser}^{65} \rightarrow \mathrm{Thr}^{65}$ (S65T), was found to accelerate the speed of fluorophore formation (from a time constant of 2 hours to 0.45 hour) (10), and several mutations located throughout the protein were found to help the molecule fold correctly at $37^{\circ} \mathrm{C}(2)$. The protein expression was improved by converting wild-type GFP (wtGFP) codons to forms more efficiently used by the organisms of interest (2), and GFP dimerization at high concentrations was overcome by the mutations $\mathrm{Ala}^{206} \rightarrow \mathrm{Lys}^{206}$, $\mathrm{Leu}^{221} \rightarrow \mathrm{Lys}^{221}$, or $\mathrm{Phe}^{223} \rightarrow \operatorname{Arg}^{223}(11)$. Finally, the major and minor absorbance peaks of wtGFP (Fig. 1C, blue open circles)
A

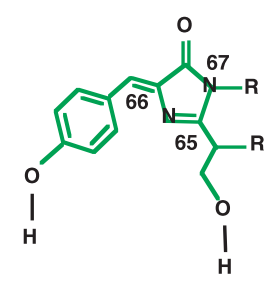

B

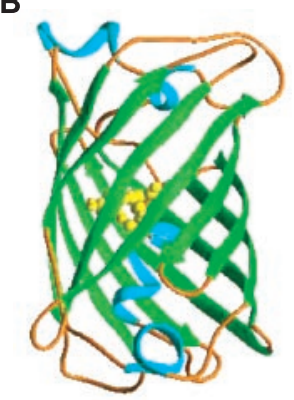

were converted to a single absorbance peak at $\sim 489 \mathrm{~nm}$ (blue squares) by substitution of the serine at the 65 position with a threonine, alanine, glycine, cysteine, or leucine $(10,12)$. This produced brighter molecules that retained a wild-type-like fluorescence (green spectra in Fig. 1C) (13).

These improved characteristics are combined in the GFP variant known as enhanced GFP (EGFP). It contains the codon optimization that enhances GFP expression in mammalian cells (2), the S65T mutation that makes an exceptionally bright, stable mutant (10), and the $\mathrm{Phe}^{64} \rightarrow \mathrm{Leu}^{64}$ mutation that alleviates GFP's temperature sensitivity (14). Proteins tagged with EGFP can be visualized in cells with low light intensities over many hours with little photobleaching (the photoinduced destruction of a fluorophore), permitting intracellular protein pathways (see movie S1) and organelle and cytoskeletal dynamics to be analyzed in unparalleled detail (15). The increased stability and brightness of the EGFP also enable the intracellular fluorescent signal from chimeras to be correlated to standard GFP solutions, permitting the quantification of molecules visualized within cells $(16,17)$.

Further developments of GFP provided variants with differing absorbance and emission spectra, allowing the simultaneous visualization of distinct GFP variants in a cell (Fig. 2, A and $\mathrm{B}$; table S1). An early screen of $A$. victoria mutants (18) produced the blue fluorescent (BFP) and cyan fluorescent (CFP) proteins. BFP has a substitution of $\mathrm{Tyr}^{66}$ with a histidine that shifts the absorbance spectrum to a peak of

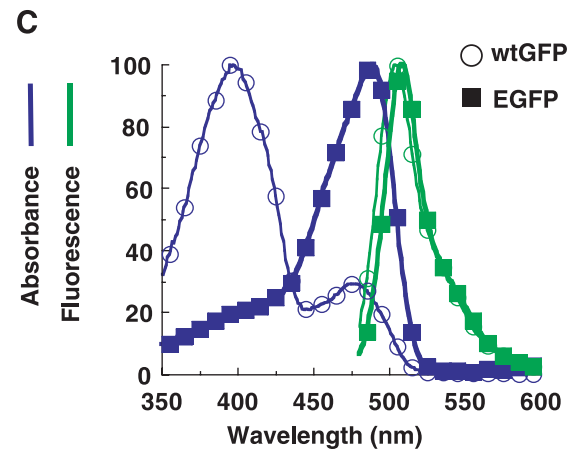

Fig. 1. (A) The wtGFP chromophore, consisting of a cyclized tripeptide made of $\mathrm{Ser}^{65}$, Tyr ${ }^{66}$, and Gly ${ }^{67}$. (B) Chromophore location within an $\alpha$ helix inside the 11-strand $\beta$ barrel of GFP [adapted from (59)]. (C) wtGFP's major and minor absorbance peaks (blue circles) and single fluorescence emission peak (green circles) compared with EGFP's single absorbance peak (blue squares) and single fluorescence emission peak (green squares). 
$384 \mathrm{~nm}$ with emission at $448 \mathrm{~nm}$ (18). Because its absorbance characteristics are readily distinguishable from EGFP, BFP was one of the first used in multicolor imaging $(19,20)$ and fluorescence resonance energy transfer (FRET) experiments [reviewed in $(2,3)]$. However, the blue variants are $\operatorname{dim}(13,20)$ and tend to photobleach readily (21); therefore, alternative multicolor pairs were developed. Of these, the most popular are cyan fluorescent protein (CFP) (18) and the red-shifted yellow fluorescent protein (YFP) (22). CFP has spectra intermediate between BFP and EGFP due to $\mathrm{Tyr}^{66}$ $\rightarrow \operatorname{Trp}^{66}$ substitution $(2,18)$ (Fig. 2, A and B). It is also brighter (20) and displays more photostability under imaging than BFP (21). Its imaging partner, YFP, was rationally designed on the basis of the GFP crystal structure to red-shift the absorbance and emission spectra with respect to EGFP and other green fluorescent variants. Efficiently excited with the 514$\mathrm{nm}$ line of an argon ion laser, YFP is much brighter than EGFP but is more sensitive to low $\mathrm{pH}$ and high halide concentrations.

The pairing of CFP-YFP has been instrumental in the study of protein-protein FRET experiments and in development of FRETbased fluorescent protein biosensors [reviewed in $(3,4)]$. Their use in the study of multiprotein trafficking has been equally important because they permit the simultaneous temporal and spatial behavior of different molecules to be analyzed [reviewed in (15)].

Efforts to further red-shift $A$. victoria fluorescent protein spectra to produce further partners for multispectral experiments have had little success. Instead, discoveries from other marine organisms have extended available fluorescent proteins to the red wavelength range (23). Of these, the DsRed (Fig. 2, A and B) from Discosoma (24), DsRed mutants (25, 26), and HcRed from Heteractis crispa (27), each with emission peaks ranging from 579 to $645 \mathrm{~nm}$, have provided the most suitable red markers (table S1). Still under improvement, the new red fluorescent proteins extend the spectrum of fluorescent tags, making multispectral imaging very feasible.

\section{Kinetic Microscopy Using Fluorescent Protein Chimeras}

New and updated fluorescence imaging methods have been essential to studies examining the localization and kinetic behavior of GFP-tagged proteins. Notable among these techniques are 4D microscopy, fluorescence recovery after photobleaching (FRAP), fluorescence loss in photobleaching (FLIP), and fluorescence correlation spectroscopy (FCS). In 4D microscopy, time-lapse observations of fluorescent molecules are collected as threedimensional data sets rather than as one image in a single focal plane (28). The enormous amounts of collected data can be interpreted with computer-based visualization programs that allow quantification and discrimination of fluorescence signals (29-31). In this way, information from the entire cell can be analyzed to provide spatial and temporal information about changes in a protein's distribution as it relates to complex cellular processes over time (32).

Although time-lapse imaging can provide information about the steady-state distribution of a protein over time, it does not reveal the kinetic properties of a molecule, for example, whether the protein is immobilized to a scaffold, free to diffuse, or undergoing constant exchange between compartments. To obtain such infor-
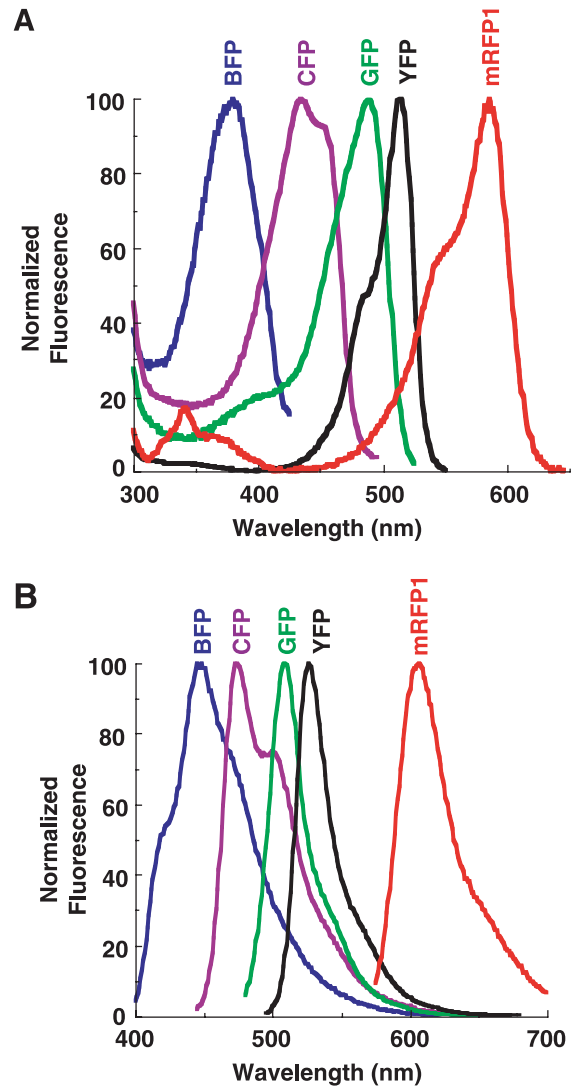

Fig. 2. The excitation (A) and emission (B) spectra of blue (BFP), cyan (CFP), green (GFP), yellow (YFP), and red (mRFP1) fluorescent proteins.

mation, a selected pool of fluorescent proteins must be distinguished from other fluorescent proteins in the cell and monitored as the two pools reequilibrate. This can be accomplished with the use of FRAP, in which an area of the cell is photobleached with a high-intensity laser pulse and the movement of unbleached molecules from neighboring areas into the bleached area is recorded by time-lapse microscopy ( 1 , 33-35) (Fig. 3A). By making invisible a fraction of the fluorescently labeled molecules in this manner, FRAP alters the fluorescence steady state in a cell without disrupting protein pathways or creating protein gradients.
Quantitative studies with fluorescent protein chimeras using FRAP can provide an estimation of the effective diffusion coefficient $\left(D_{\text {eff }}\right)$ and mobile fraction $\left(M_{\mathrm{f}}\right)$ of a protein $(36-38)$. The $D_{\text {eff }}$ reflects the mean squared displacement that an idealized protein moves by random walk over time and is inversely proportional to the size of the protein. Because most proteins readily interact with other molecules in cells, the $D_{\text {eff }}$ of a protein measured using FRAP must be carefully interpreted $(1,34,35)$. For example, when the diffusion rate is significantly lower than what is predicted based on the protein's size, the protein could be incorporated into an aggregate or a large complex. Alternatively, the environment of a protein could be significantly more viscous than expected, or the protein could be interacting transiently with a fixed substrate. By contrast, if the mobility of a protein is faster than predicted, the protein might be showing directed transport by motor proteins or the viscosity of the environment might be decreased. The fraction of fluorescent proteins that can diffuse into a bleached region during the time course of a FRAP experiment, or $M_{\mathrm{f}}$, also reveals important information about a protein. For example, a decrease in $M_{\mathrm{f}}$ indicates that the protein could be binding to fixed molecules, forming immobile aggregates, or that the protein is confined in a separate compartment. An increase in $M_{\mathrm{f}}$, on the other hand, indicates that the protein could be released from a fixed scaffold or exported out of a discontinuous compartment.

Two other fluorescence imaging techniques, FLIP and FCS, also provide information about a protein's kinetic properties. FLIP is performed by repeatedly photobleaching fluorescence in one area of the cell while collecting images of the entire cell (Fig. 3B) (1). By monitoring the fluorescence in the nonphotobleached regions, the mobility of a fluorescently tagged protein can readily be observed and the continuity of cellular environments determined $(36,38,39)$. In FCS, fluorescently labeled molecules diffusing in and out of a small defined focal volume $(\sim 1$ femtoliter) are measured over short periods of time (40). The fluctuations in molecules moving through the focal volume can be analyzed to obtain a protein's diffusion coefficient, binding constants, and concentrations $(41,42)$. FCS holds great promise for analyzing the dynamic properties of fluorescent protein chimeras in living cells.

Computational methods can be used to interpret a protein's $D_{\text {eff }}$ and $M_{\mathrm{f}}$ obtained from FRAP, FLIP, and FCS experiments through the testing of simple models (34). In this approach, a mathematical model is constructed that describes a protein's biophysical parameters, such as binding and release from a substrate, residency time in a compartment, turnover, and diffusional mobility. It is then tested with experi- 
mental data. The parameter values that best fit the data can provide important new quantitative information about a protein's dynamics in cells $(16,43,44)$. Several modeling software packages are available for testing simple models of protein behavior to quickly evaluate hypotheses or to interpret experimental data, including SAAMII, Berkley Madonna, and MatLAB (34). In addition, highly integrated software programs such as the Virtual Cell (45) allow the building of larger models to test the predicted behavior of more complex, nonlinear systems such as signaling networks.

Results from kinetic microscopy experiments using GFP fusion proteins and computational methods have revealed many important properties of cells unattainable by traditional in vitro biochemical methods. These include the dynamics of large macromolecular complexes in the cytoplasm (46) and nucleus $(43,47)$; the rate of exchange on and off membranes of cytoplasmic proteins $(44,48)$; the residency time and diffusion coefficients of proteins in different compartments (16, $36)$; and the characterization of the viscous properties of the cytoplasm, organelle lumens, and cell membranes $(38,39,49)$.

\section{Destabilized GFP \\ Variants and \\ Photo-Modulatable Fluorescent Proteins}

Measurement of protein turnover or analysis of the temporal expression pattern and behavior of proteins is difficult, if not impossible, with conventional GFP variants. This is because the GFP chimeras are continuously being synthe-

\section{A Fluorescence Recovery After Photobleaching (FRAP)}

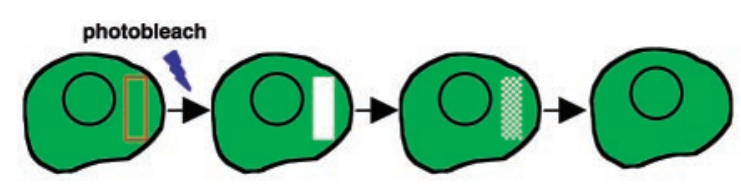

B

$$
\text { Fluorescence Loss in Photobleaching (FLIP) }
$$

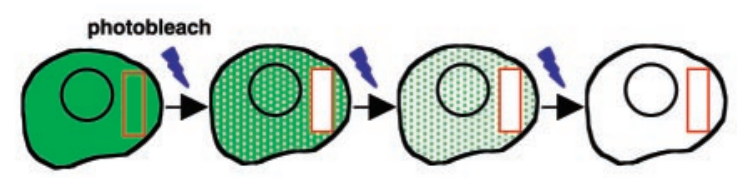

C
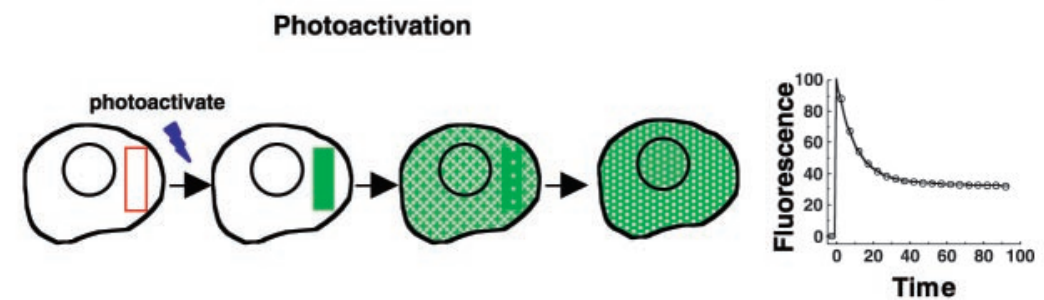

Fig. 3. Kinetic microscopy techniques. (A) In FRAP, a region of the cell (indicated in red) is selectively and intensely irradiated to photobleach fluorescent molecules. The recovery of fluorescent molecules into that region is assessed quantitatively to determine diffusion coefficients and mobile fractions. (B) In FLIP, a region of the cell (indicated in red) is repeatedly photobleached. Movement of fluorescent molecules into the region being photobleached results in loss of fluorescence from areas outside the box and can be used to access the boundaries for a protein's diffusional movement within a cell. (C) In photoactivation, selective irradiation of region (indicated in red) leads to the molecules in this region becoming fluorescent. These molecules can then be monitored as they move out of this region and throughout the cell.

\section{sized, folded, and de-} graded within cells. At any particular time, therefore, proteins at different stages of their lifetime are being observed. One approach to characterize a protein's expression timing or lifetime is to use destabilized variants of GFP (50). By creating a GFP that is rapidly turned over by proteolysis, younger protein chimeras can be distinguished from older chimeras that have lost their fluorescence due to GFP degradation. Of course, the rapid degradation problematically maintains a low fluorescence signal. A different approach is to use fluores- of photoactivable fluorescent proteins. These proteins display little initial fluorescence under excitation at the imaging wavelength but increase their fluorescence after activation by irradiation at a different wavelength (Fig. $3 C)$. This results is the direct highlighting of distinct pools of molecules within the cell. Because only photoactivated molecules exhibit noticeable fluorescence, their lifetime and behavior can be studied independently of other newly synthesized proteins.

Early efforts (52-54) in the production of a photoactivatable fluorescent protein had relatively modest success ( $\leq$ threefold photoactivation). Recently, three molecules-PAGFP (5), Kaede (6), and KFP1 (7)-have been shown to display $\geq 30$-fold increases in fluorescence after photoactivation. PA-GFP was developed by improving on the wtGFP photoconversion from a neutral to anionic species (5) [Supporting Online Material (SOM) Text]. In the context of a wtGFP chromophore, several mutations at the $\mathrm{Thr}^{203}$ position $(5,18,55)$ decrease the initial absorbance at $\sim 475 \mathrm{~nm}$ (compare Fig. 4, A and C) while still permitting photoconversion (5) (Fig. 4, B and D). The $\mathrm{Thr}^{203} \rightarrow \mathrm{His}^{203}$ mutant (named PA-GFP) exhibits up to 100 -fold increases in fluorescence excitation at $488 \mathrm{~nm}$ when illuminated with 413-nm light (5). Kaede (6), on the other hand, was identified from the stony coral Trachyphyllia geoffroyi. Irradiation of Kaede at $\sim 400 \mathrm{~nm}$ results in a spectral shift from peaks at $508 \mathrm{~nm}$ (absorbance) and $518 \mathrm{~nm}$ (emission) to $572 \mathrm{~nm}$ and $582 \mathrm{~nm}$, respectively. These shifts result in a striking $\sim 2000$-fold increase in Kaede's red-togreen fluorescence ratio. Lastly, improvements to asCP (asFP595) from the sea anemone, Anemonia sulcata, resulted in a mutant denoted KFP1 that displays $\sim 30$-fold increase in red fluorescence upon irradiation with green light (7).

Although Kaede displays the largest contrast between pre- and postphotoactivation (2000fold) and is therefore the best choice for marking single cells within a population, both it and KFP1 self-associate to form tetramers. This makes them problematic as fusion tags, unlike the A. victoria-derived PAGFP whose self-association is weak (11) and which can be used as a reliable protein reporter (5). But, photoactivation of KFP1 with light of $\sim 532 \mathrm{~nm}$ (7) is likely to be less harmful to cells than the near-ultraviolet light of $\sim 400 \mathrm{~nm}$ required to photoactivate PAGFP and Kaede $(5,6)$.

In addition to their potential use as expression and degradation timers, PA-GFP, Kaede, and KFP1 can be used to complement other kinetic microscopy techniques for monitoring 
protein behavior in living cells. The short time required for photoactivation (in some cases, $<1 \mathrm{~s}$ ) makes it potentially superior to photobleaching as a method for examining the diffusional mobility and compartmental residency time of proteins (Fig. 4E) (5). Because only photoactivated proteins are fluorescent within the cell, studies of the turnover and degradative pathway of proteins can be readily studied. Photoactivatable proteins are also useful as expression markers to follow the behavior of subsets of cells within a larger population (6) or in cell lineage studies (7).

\section{Looking to the Future}

Continued efforts to engineer new fluoroexpanding the uses of fluorescent probes in the investigation of complex cellular processes. Brighter and more red-shifted fluorescent proteins, for example, can provide additional tags for multispectral imaging and FRETbased methods and serve as probes for greater phores and reporter classes is important for

tissue penetration. Photoactivatable variants with different spectral properties are needed for simultaneous tracking of two or more types of photoactivated proteins. Fluorophores with increased brightness can help in studies tracking single molecules, whereas ones with more $\mathrm{pH}$ resistance will be useful for probing acidic cellular environments. The $>25$ fluorescent proteins isolated from different marine organisms (23) could help satisfy these needs once these proteins are further characterized and developed.

Advances in modern imaging systems are also important for keeping pace with these developments in fluorescent labeling. More sensitive and quicker camera systems, filter systems for optimally detecting different fluorophores, superior software for quantifying and discriminating fluorescent signals, and hardware for photobleaching and photoactivating geometrically defined spatial patterns are desirable. Recent advances in microscopy imaging tech-

A
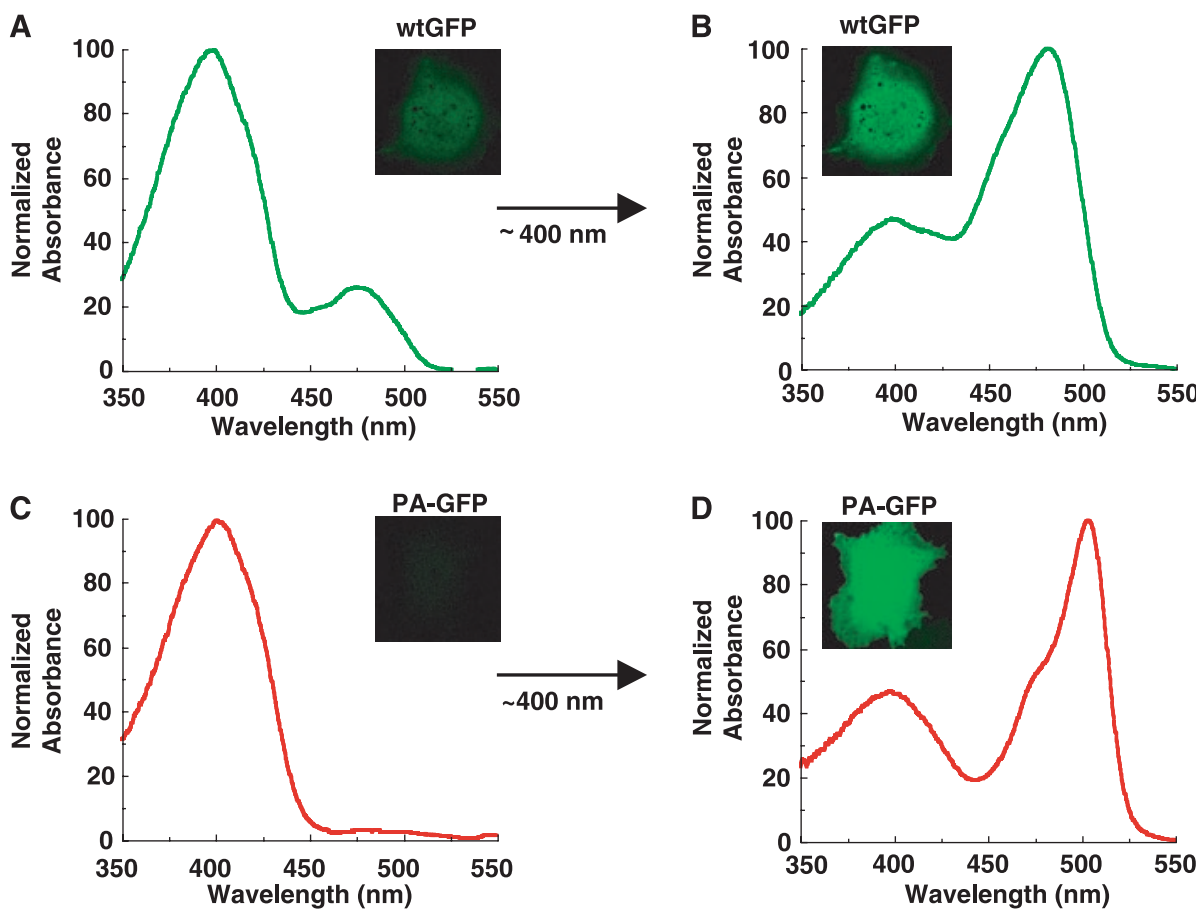

E
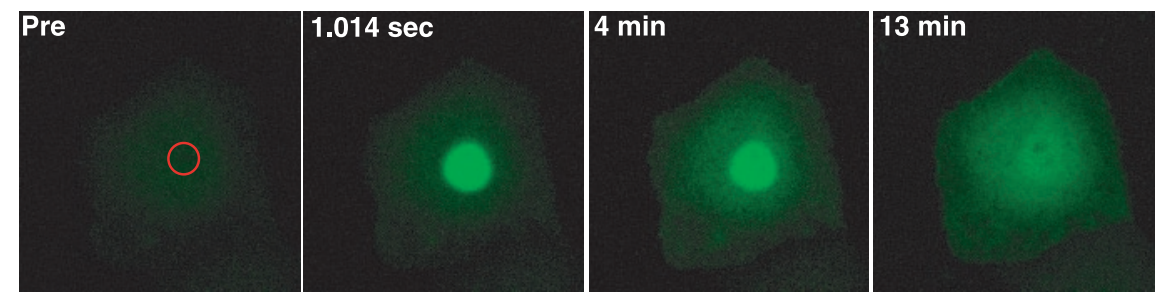

Fig. 4. Photoactivatable GFP. Absorbance spectra of purified wtGFP before (A) and after (B) photoactivation with $\sim 400$-nm light. The inset images show the 488-nm excited fluorescence from a cell expressing wtGFP before (A) and after (B) photoactivation. Absorbance spectra of purified PA-GFP before (C) and after (D) photoactivation with 400-nm light. The inset images show the 488-nm excited fluorescence from a cell expressing PA-GFP before (C) and after (D) photoactivation. (E) A cell expressing PA-GFP was imaged with the use of 488-nm excitation (Pre) before and after $\sim 1$-s photoactivation of the nuclear pool with 413-nm laser light. niques, including two photon (56), total internal reflection (57), and stimulated emission depletion (58), can provide additional ways to visualize and quantify molecules and events with high spatial and temporal resolution. Finally, understanding the complex protein interactions and dynamics generated from these methods will require the use of kinetic modeling and analysis tools.

\section{References and Notes}

1. J. Lippincott-Schwartz, E. Snapp, A. Kenworthy, Nature Rev. Mol. Cell Biol. 2, 444 (2001).

2. R. Y. Tsien, Annu. Rev. Biochem. 67, 509 (1998)

3. P. van Roessel, A. H. Brand, Nature Cell Biol. 4, E15 (2002).

4. J. Zhang, R. E. Campbell, A. Y. Ting, R. Y. Tsien, Nature Rev. Mol. Cell Biol. 3, 906 (2002).

5. G. H. Patterson, J. Lippincott-Schwartz, Science 297, 1873 (2002).

6. R. Ando, H. Hama, M. Yamamoto-Hino, H. Mizuno, A. Miyawaki, Proc. Natl. Acad. Sci. U.S.A. 99, 12651 (2002).

7. D. M. Chudakov et al., Nature Biotechnol. 21, 191 (2003).

8. D. C. Prasher, V. K. Eckenrode, W. W. Ward, F. G. Prendergast, M. J. Cormier, Gene 111, 229 (1992).

9. M. Chalfie, Y. Tu, G. Euskirchen, W. W. Ward, D. C. Prasher, Science 263, 802 (1994).

10. R. Heim, A. B. Cubitt, R. Y. Tsien, Nature 373, 663 (1995).

11. D. A. Zacharias, J. D. Violin, A. C. Newton, R. Tsien, Science 296, 913 (2002).

12. S. Delagrave, R. E. Hawtin, C. M. Silva, M. M. Yang, D. C. Youvan, Bio-Technology 13, 151 (1995).

13. A. B. Cubitt, L. A. Woollenweber, R. Heim, in Green Fluorescent Proteins, K. F. Sullivan, S. A. Kay, Eds. (Academic Press, San Diego, CA, 1997), vol. 58, p. 19.

14. B. P. Cormack, R. H. Valdivia, S. Falkow, Gene 173, 33 (1996).

15. J. Lippincott-Schwartz, T. H. Roberts, K. Hirschberg, Annu. Rev. Cell Dev. Biol. 16, 557 (2000).

16. K. Hirschberg et al., J. Cell Biol. 143, 1485 (1998).

17. D. W. Piston, G. H. Patterson, S. M. Knobel, in Green Fluorescent Proteins, K. F. Sullivan, S. A. Kay, Eds. (Academic Press, San Diego, CA, 1999), vol. 58, p. 31.

18. R. Heim, D. C. Prasher, R. Y. Tsien, Proc. Natl. Acad. Sci. U.S.A. 91, 12501 (1994)

19. R. Rizzuto et al., Curr. Biol. 6, 183 (1996).

20. R. Heim, R. Y. Tsien, Curr. Biol. 6, 178 (1996).

21. J. Ellenberg, J. Lippincott-Schwartz, J. F. Presley, Biotechniques 25, 838 (1998).

22. M. Ormö et al., Science 273, 1392 (1996).

23. Y. A. Labas et al., Proc. Natl. Acad. Sci. U.S.A. 99, 4256 (2002).

24. M. V. Matz et al., Nature Biotechnol. 17, 969 (1999).

25. B. J. Bevis, B. S. Glick, Nature Biotechnol. 20, 83 (2002).

26. R. E. Campbell et al., Proc. Natl. Acad. Sci. U.S.A. 99, 7877 (2002).

27. N. G. Gurskaya et al., FEBS Lett. 507, 16 (2001).

28. D. Gerlich, J. Beaudouin, M. Gebhard, J. Ellenberg, R. Eils, Nature Cell Biol. 3, 852 (2001).

29. H. Bornfleth, P. Edelmann, D. Zink, T. Cremer, C. Cremer, Biophys. J. 77, 2871 (1999).

30. W. Tvaruskó et al., Proc. Natl. Acad. Sci. U.S.A. 96, 7950 (1999).

31. C. B. J. Bergsma, G. J. Streekstra, A. W. M. Smeulders, E. M. M. Manders, Cytometry 43, 261 (2001).

32. A. T. Hammond, B. S. Glick, Traffic 1, 935 (2000).

33. D. Axelrod, D. E. Koppel, J. Schlessinger, E. Elson, W. W. Webb, Biophys. J. 16, 1055 (1976)

34. R. D. Phair, T. Misteli, Nature Rev. Mol. Cell Biol. 2, 898 (2001).

35. E. A. J. Reits, J. J. Neefjes, Nature Cell Biol. 3, E145 (2001).

36. N. B. Cole et al., Science 273, 797 (1996).

37. R. Swaminathan, C. P. Hoang, A. S. Verkman, Biophys. J. 72, 1900 (1997).

38. S. Nehls et al., Nature Cell Biol. 2, 288 (2000). 
39. M. J. Dayel, E. F. Y. Hom, A. S. Verkman, Biophys. J. 76, 2843 (1999).

40. E. Van Craenbroeck, Y. Engelborghs, J. Mol. Recognition 13, 93 (2000)

41. P. Schwille, U. Haupts, S. Maiti, W. W. Webb, Biophys. J. 77, 2251 (1999)

42. R. Brock, G. Vamosi, G. Vereb, T. M. Jovin, Proc. Natl. Acad. Sci. U.S.A. 96, 10123 (1999).

43. R. D. Phair, T. Misteli, Nature 404, 604 (2000)

44. J. F. Presley et al., Nature 417, 187 (2002).

45. I. I. Moraru, J. C. Schaff, B. M. Slepchenko, L. M. Loew, Ann. N. Y. Acad. Sci. 971, 595 (2002).

46. A. V. Nikonov, E. L. Snapp, J. Lippincott-Schwartz, G Kreibich, J. Cell Biol. 158, 497 (2002)

47. J. G. McNally, W. G. Müller, D. Walker, R. Wolford, G. L. Hager, Science 287, 1262 (2000).

48. X. Wu et al., J. Cell Biol. 155, 291 (2001)
49. A. Partikian, B. Ölveczky, R. Swaminathan, Y. Li, A. S. Verkman, J. Cell Biol. 140, 821 (1998)

50. X. Li et al., J. Biol. Chem. 273, 34970 (1998).

51. A. Terskikh et al., Science 290, 1585 (2000).

52. H. Yokoe, T. Meyer, Nature Biotechnol. 14, 1252 (1996)

53. K. A. Lukyanov et al., J. Biol. Chem. 275, 25879 (2000)

54. J. S. Marchant, G. E. Stutzmann, M. A. Leissring, F. M. LaFerla, I. Parker, Nature Biotechnol. 19, 645 (2001).

55. T. Ehrig, D. J. O'Kane, F. G. Prendergast, FEBS Lett. 367, 163 (1995).

56. W. Denk, D. W. Piston, W. W. Webb, in Handbook of Biological Confocal Microscopy, J. B. Pawley, Ed. (Plenum Press, New York, 1995), p. 445.

57. J. A. Steyer, W. Almers, Nature Rev. Mol. Cell Biol. 2 268 (2001).
58. M. Dyba, S. W. Hell, Phys. Rev. Lett. 88, 163901 (2002).

59. F. Yang, L. G. Moss, G. N. Phillips Jr., Nature Biotechnol. 14, 1246 (1996).

60. We thank D. Piston (Vanderbilt University) for Histagged EBFP, ECFP, EGFP, and EYFP protein expression plasmids and R. Tsien (Howard Hughes Medical Institute, University of California San Diego) for the His-tagged mRFP1 protein expression plasmid.

Supporting Online Material

www.sciencemag.org/cgi/content/full/300/5616/87/DC1 SOM Text

Fig. S1

Table S1

References and Notes

Movie S1

\title{
Mitosis Through the Microscope: Advances in Seeing Inside Live Dividing Cells
}

\author{
Conly L. Rieder* and Alexey Khodjakov*
}

\begin{abstract}
The most visually spectacular events in the life of a cell occur when it divides. This is especially true in higher eukaryotes, where the size and geometry of cells allow the division process to be followed through a microscope with considerable clarity. In these organisms, the membrane surrounding the nucleus breaks down after the replicated DNA has condensed to form discrete chromosomes. Several new structures are then assembled to separate the chromosomes and partition the cytoplasm into two separate cells.
\end{abstract}

The German anatomist Walther Flemming was one of the first to describe the cell division process (1). In 1882 he coined the term "mitosis" to characterize the formation of paired threads (Greek $=$ mitos) during division of the cell nucleus (Fig. 1). These threads, which formed from a substance Flemming called chromatin, came to be known as the "chromosomes." The definition of mitosis has since been expanded to include "cytokinesis," the process by which the cell cytoplasm is partitioned at the end of nuclear division.

Until the late 1940 s, research on mitosis was primarily restricted to an examination of cells that had been preserved in a lifelike state by chemicals (i.e., fixed) and then colored with dyes to generate contrast between their different components (2). These descriptions revealed that the division process is fundamentally the same in all somatic cells. In animals, mitosis is mediated by a bipolar, spindle-shaped apparatus that

Lab of Cell Regulation, Division of Molecular Medicine, Wadsworth Center, Albany, NY 12201-0509, USA. Department of Biomedical Sciences, State University of New York, Albany, NY 12222, USA. Marine Biology Laboratory, Woods Hole, MA 02543, USA.

*To whom correspondence should be addressed. Molecular Medicine, Wadsworth Center, Post Office Box 509, Albany, NY 12201-0509, USA. E-mail: rieder@ wadsworth.org, khodj@wadsworth.org appears to be assembled in the cytoplasm from two radial arrays of fibers, known as "asters." These asters form in association with two separating "centrosomes" that define the spindle poles (Fig. 1, E and F). Early studies also noted that each chromosome possesses two small organelles on its surface that are positioned back-to-back and on opposite sides of the chromosome. As the spindle forms, these "kinetochores" acquire fibers that attach them to one of the spindle poles, so that opposing sister kinetochores are attached to opposite poles (Fig. $1 \mathrm{~J})$. Collectively, the spindle and its associated centrosomes, kinetochores, and chromosomes are referred to as the mitotic apparatus.

Flemming noted that the chromosomes, which are scattered throughout the cytoplasm after nuclear envelope breakdown (Fig. 1D), are collected by the spindle and positioned on a plane halfway between the two poles (Fig. 1F). After this "metaphase" alignment is completed, the two chromatids forming each chromosome disjoin, and each moves toward its respective pole in a process termed "anaphase" (Fig. 1, G and H). Once the two groups of chromosomes reach their respective poles, they coalesce to form the new daughter nuclei, after which cytokinesis pinches the cytoplasm into two new cells (Fig. 1I).
Considering that $\sim 2.5 \times 10^{8}$ cells are dividing in the human body at any given time (3), even if few errors occur, many genetically abnormal cells will be produced during the lifetime of an organism. Some of these will lose their ability to regulate the cell cycle, which is one of the attributes of cancer cells (4). An important goal of cancer research is, therefore, to define the molecular mechanisms that form the spindle and generate the forces to move the chromosomes. A more recent focus is to understand how the cell regulates progression through the division process. Surprisingly, these problems are intimately linked because chromosome motion and progression through mitosis are both governed by the formation of kinetochore fibers.

As the description of a cellular event becomes more accurate, the corresponding molecular model(s) become more meaningful. Because mitosis involves many concurrent visible events, advances in understanding the mechanisms involved are historically linked to technological advances in light microscopy. What follows is a brief and roughly chronological review of these advances, with selected examples of how they have progressively refined our understanding of mitosis in higher animal cells (5).

\section{Observing the Behavior of the Spindle and Chromosomes in Living Cells}

The development of cell culture methods in the 1920 s set the stage for studies on how living vertebrate cells divide. Early observations were hampered, however, because cellular components are not naturally contrasted when viewed with traditional bright-field optics (Fig. 2A). This situation changed radically in the mid 1950s 